\title{
Valuation of inventories in systems with product recovery
}

\author{
Ruud Teunter* $\quad$ Erwin van der Laan $^{\dagger}$
}

August 29, 2003

Econometric Institute Report EI2003-29

\section{Introduction}

Valuation of inventories has different purposes, in particular accounting and decision making, and it is not necessary for a firm to use the same valuation method for both purposes. In fact, it is not uncommon to use accounting books as well as management books. In this chapter, we will only consider inventory values from the perspective of decision making. More specifically, we will analyze the effect of inventory valuation on inventory control decisions (and not the corresponding financial results) for systems with product recovery. Of course, inventory valuation also influences other strategic and operations management decisions concerning product recovery, as is illustrated by the following real-life example.

*Erasmus University Rotterdam, Econometric Institute, Burg. Oudlaan 50, PO Box 800, 3000 DR Rotterdam, The Netherlands. E-mail: teunter@few.eur.nl

${ }^{\dagger}$ rasmus University Rotterdam, Department of Decision and Information Sciences, Burg. Oudlaan 50, PO Box 800, 3000 DR Rotterdam, The Netherlands. E-mail: elaan@fac.fbk.eur.nl 


\section{Case: Product recovery of copiers}

A copier producer/remanufacturer has a single European (re)manufacturing facility $(\mathrm{CF})$ and a number of National sales/lease Organizations (NO). The NO operate independently, purchasing new and/or remanufactured copiers from the $\mathrm{CF}$ and returning used copiers to the CF. The product flows are controlled by the CF using internal purchase prices and return fees. The return fees for used copiers and the purchase prices for remanufactured copiers are based on the valuation by the CF of used and remanufactured copiers. Using different valuation methods over the last few years has lead to some important insights. Higher return fees lead to more returns of newer models. Though this implies more recovery opportunities, there is also the associated risk of NO returning copiers that can still be leased/sold at a reasonable profit. As expected, higher purchase prices for remanufactured copiers lead to less demand from the NO. Lower values for used and remanufactured copiers indicate that remanufacturing is less expensive than production, motivating designers to build 'green' copiers.

But our focus is solely on inventory control decisions. We start by describing the link between inventory values and inventory control decisions. This discussion is not restricted to systems with product recovery. In fact, it is based on literature for systems without product recovery.

Both in the modeling theory and in practice, inventory control decisions are often based on an average cost $(A C)$ model of reality. Instead of analyzing the effect of inventory decisions on cash flows, the AC approach transforms cash flows into costs because those are easier to work with. Consider, for instance, the purchase of a product at price $c$ at time $t$. Assume that this product is kept in stock until a demand occurs at time $T \geq t$. The associated purchase cash flow, discounted at rate $\alpha$ to time $T$, is $c e^{\alpha(T-t)} \approx c+c \alpha(T-t)$. The AC approach transforms this cash flow into a purchase cost $c$ at time $t$ and a holding cost $h(T-t)$ during period $(t, T)$, where $h$ is the so-called opportunity holding cost rate. Clearly, for this example, setting $h=c \alpha$ approximately transforms the discounted cash flow $(D C F)$ into costs.

This simple example illustrates that the inventory holding cost rates are crucial in that transformation process. The AC approach adds an opportunity (non-cash flow) holding cost rate to the 'true' out-of-pocket (cash flow) holding cost rate. The opportunity holding cost rate is generally calculated by multiplying the value of a product with the discount/interest rate (see also the above example). See, for instance, Naddor (1966), Silveret al. (1998), and Tersine (1988). So, in AC approaches, product values influence inventory control decisions via holding cost rates.

For many models with forward logistics only, it has been shown that the approximate AC approach with holding cost rates calculated as described above leads to (nearly) the same inventory control decisions as the exact DCF approach (see Klein Haneveld and Teunter, 1998, and Corbey et al.,1999). But does the same hold for models with product recovery? And if so, how do we determine the 'right' values for recoverable and recovered 
products? These questions were avoided in Chapters 7 to 10, where many AC models of inventory systems with product recovery were analyzed. There, the holding cost rates were simply assumed to be given, without referring to the underlying cash flows. In this chapter, we will deal with the above questions. To keep the discussion and analysis transparent, we do not consider out-of-pocket holding costs, so costs related to inventory investment are assumed to be 'opportunity costs' only. We remark, however, that out-ofpocket holding costs can easily be included in an AC approach by adding the out-of-pocket holding cost rate to the opportunity holding cost rate.

The remainder of this chapter, is organized as follows. In Section 2, we review those studies that compare the AC model and the DCF model analytically. All consider the simple inventory system that is depicted graphically in Figure 1. (Some studies include the 'dotted' disposal option, but others do not.)

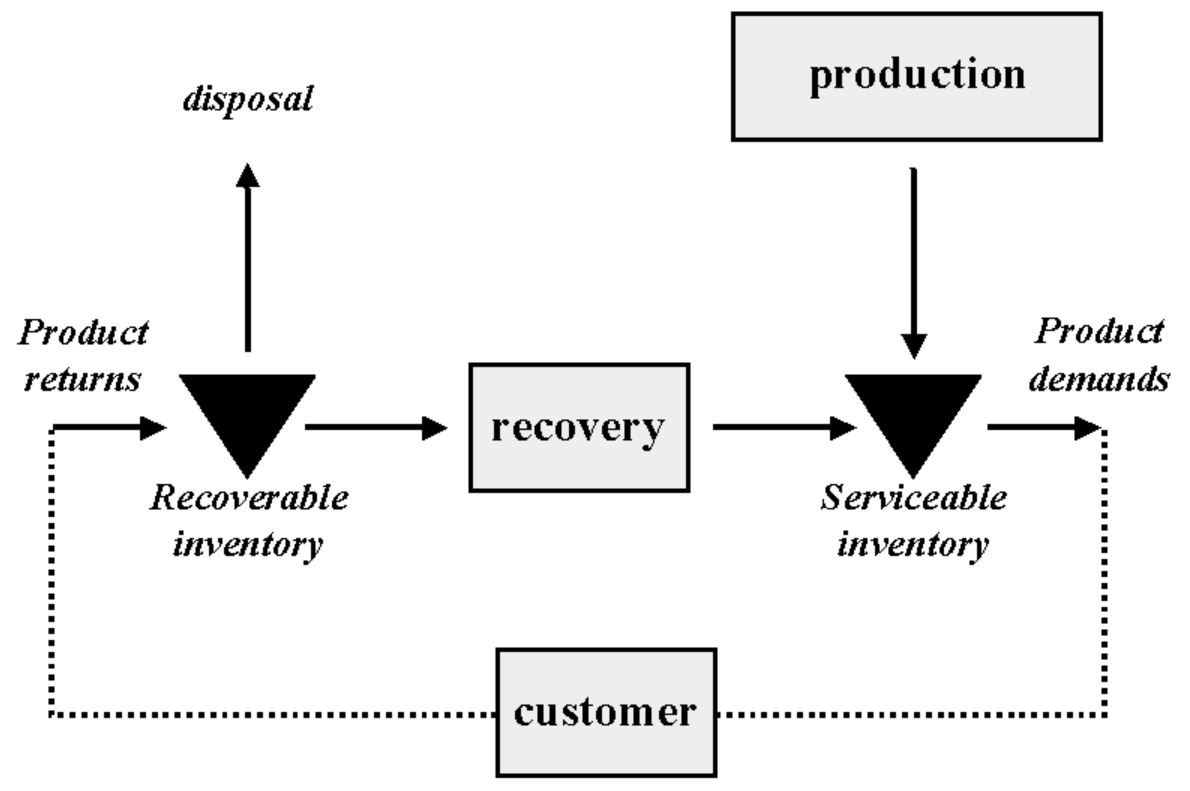

Figure 1: A simple inventory system with product recovery

The results of these studies show that there indeed exist values (and corresponding holding cost rates) that approximately transform the $\mathrm{AC}$ into the DCF. Some of those values are surprising, in the sense that they would not result from the traditional 'cost price reasoning'. In Section 3, we discuss a simulation study (of a model based on the inventory systems depicted in Figure 1) with similar findings, and offer intuitive explanations. Moreover, based on those explanations, we discuss the conditions under which we expect certain values to approximately transform AC into DCF. In Section 3, this discussion is restricted to the inventory system in Figure 1, but in the following section we also 


\begin{tabular}{l|l}
$d$ & demand rate \\
$u$ & return rate \\
$\alpha$ & discount rate \\
$c_{p}$ & production cost (per product) \\
$c_{r}$ & remanufacturing cost (per product) \\
$c_{w}$ & disposal cost (per product) \\
$K_{p}$ & set-up cost for production \\
$K_{r}$ & set-up cost for remanufacturing \\
$K_{w}$ & set-up cost for disposal \\
$A C$ & average cost \\
$N P V$ & net present value \\
$A S$ & annuity stream $\alpha N P V$ \\
$Q_{p}$ & production order quantity \\
$Q_{r}$ & remanufacturing order quantity \\
$h_{s}$ & holding cost rate for serviceable products \\
$h_{r}$ & holding cost rate for remanufacturable returned products \\
$h_{w}$ & holding cost rate for disposable returned products
\end{tabular}

Table 1: Notations

consider more complex inventory systems with (dis)assembly of (returned) products. In Section 5, we discuss the implications of these findings for systems without recovery but with multiple sources for obtaining serviceable products. We end with conclusions and directions for future research in Section 6.

Throughout this chapter, we use the notations listed in Table 1.

\section{Analytical comparisons of average cost and dis- counted cash flow}

\subsection{Discounted Cash Flow, Net Present Value, and Annuity Stream}

Consider a series of deterministic cash flows $C_{1}, C_{2}, \ldots, C_{N}$ at times $T_{1}, T_{2}, \ldots, T_{N}$. The total discounted cash flow, i.e. the net present value $(N P V)$, of this series can be calculated as

$$
\sum_{n=1}^{N} C_{n} \mathrm{e}^{-\alpha T_{n}} .
$$

For stochastic systems, let us use the $N P V$ as the expectation of the total discounted cash flow over an infinite horizon. As an example, consider a cash flow $C$ that occurs at stochastic times $T_{1}, T_{2}, \ldots$ with independent inter-occurrence times, $S_{1}=T_{1}-0$ and $S_{n}:=T_{n}-T_{n-1}$ for $n>1$, with probability density function 


$$
f_{S_{n}}(t)= \begin{cases}z_{1}(t), & n=1 \\ z(t), & n>1\end{cases}
$$

Note that inter-occurrence times are identically distributed from time $T_{1}$, i.e., the cash flow is cyclic from time $T_{1}$. But the cash flow is not necessarily cyclic from time 0 , and therefore the first inter-occurrence is treated separately. Such cash flow series are basic elements that return in many of the subsequent models. The $N P V$ of this series of cash flows, discounted at rate $\alpha$, equals

$$
\begin{aligned}
N P V & =\mathrm{E}\left\{\sum_{n=1}^{\infty} C \mathrm{e}^{-\alpha T_{n}}\right\}=C \sum_{n=1}^{\infty} \int_{0}^{\infty} z_{1} * z^{*(n-1)}(t) \mathrm{e}^{-\alpha t} \mathrm{~d} t \\
& =C \tilde{z}_{1}(\alpha) \sum_{n=1}^{\infty} \tilde{z}(\alpha)^{(n-1)}=\frac{C \tilde{z}_{1}(\alpha)}{1-\tilde{z}(\alpha)}
\end{aligned}
$$

where the asterisk denotes convolution and $\tilde{z}(s)=\int_{0}^{\infty} z(t) \mathrm{e}^{-s t} \mathrm{~d} t$ denotes the Laplace transform of time function $z(t), t \geq 0$, for complex $s$.

If a stream of cash flows has net present value $X$, then a continuous cash flow of $\alpha X$ has the same net present value, i.e. $X$. Therefore, we define the so-called Annuity Stream $(A S)$ as

$$
A S=\alpha N P V
$$

The annuity stream is useful since it can be compared directly with average costs.

\subsection{A stochastic inventory model with production and instan- taneous remanufacturing}

For a first analysis, we consider the inventory system depicted in Figure 1. To keep the analysis tractable, we assume that the demand process and the return process are independent Poisson processes with rates $d$ and $u<d$ respectively. We further assume that the lead times for both manufacturing and remanufacturing are zero, and that backorders are not allowed.

There is a setup cost $K_{p}$ per production order, a variable production cost $c_{p}$ per product, and a variable remanufacturing $\operatorname{cost} c_{r}$ per product. Here $c_{r}<c_{p}$, otherwise remanufacturing would never be preferred over production.

Inventory is controlled by a continuous review PUSH strategy, which is defined as follows (see Section 8.3.2 of Chapter 8 for a detailed discussion). Remanufacturing is instantaneous, i.e. starts as soon as a product is returned. So, there is no disposal nor stocking of remanufacturables. Production occurs in batches of fixed size $Q_{p}$, and starts whenever the inventory drops below zero. Note that this is the optimal strategy structure under the above assumptions and cost structure (see Fleischmann and Kuik, 1998). The 
(serviceable) inventory level at time zero equals $I_{0}$. Under the NPV criterion all cash inand out-flows are discounted with opportunity cost rate $\alpha$.

For simplicity, we do not consider cash flows related to sales and acquisition of product returns, but they could easily be included in the analysis.

Define $\left\{R_{n} \mid n \geq 1\right\}$ and $\left\{P_{n} \mid n \geq 1\right\}$ as the occurrence times of remanufacturing orders and production orders, respectively. Note that the stream of remanufacturing orders is a Poisson process, since the return process is a Poisson process also, and all returns are instantaneously remanufactured.

Since $\sum_{n=1}^{\infty} \mathrm{E}\left(\mathrm{e}^{-\alpha R_{n}}\right)=u / \alpha$, the annuity stream as a function of order size $Q_{p}$ reads

$$
\begin{gathered}
A S\left(Q_{p}\right)=\alpha \sum_{n=1}^{\infty} \mathrm{E}\left(c_{r} \mathrm{e}^{-\alpha R_{n}}+\left(K_{p}+c_{p} Q_{p}\right) \mathrm{e}^{-\alpha P_{n}}\right) \\
=c_{r} u+\alpha\left(K_{p}+c_{p} Q_{p}\right) \frac{\tilde{f}_{I_{0}}(\alpha)}{1-\tilde{f}_{Q_{p}-1}(\alpha)},
\end{gathered}
$$

where $f_{i}(t)$ is the probability density function with regard to the first-occurrence time of production orders, given that the process starts with inventory level $i$. These functions cannot be calculated directly, so instead we develop the following procedure.

Suppose that at time zero the inventory level is $i \geq 0$. Either the next occurrence is a demand at time $t$, with probability density $g(t)=d \mathrm{e}^{-(d+u) t}$, which moves the inventory down to $i-1$, or the next occurrence is a return, with probability density $h(t)=u \mathrm{e}^{-(d+u) t}$, which moves the inventory level up to $i+1$. Thus we have

$$
f_{i}(t)= \begin{cases}g(t)+h * f_{1}(t), & i=0 \\ g * f_{i-1}(t)+h * f_{i+1}(t), & i>0 .\end{cases}
$$

Taking Laplace transforms and evaluating at $\alpha,(2)$ becomes

$$
\tilde{f}_{i}(\alpha)= \begin{cases}\tilde{g}(\alpha)+\tilde{h}(\alpha) \tilde{f}_{1}(\alpha), & i=0 \\ \tilde{g}(\alpha) \tilde{f}_{i-1}(\alpha)+\tilde{h}(\alpha) \tilde{f}_{i+1}(\alpha), & i>0\end{cases}
$$

where $\tilde{g}(\alpha)=\frac{d}{d+u+\alpha}$ and $\tilde{h}(\alpha)=\frac{u}{d+u+\alpha}$ are the Laplace transforms of $g(t)$ and $h(t)$ respectively, and $\tilde{f}_{i}(\alpha)$ denotes the discounted first occurrence time of a production order, given that the inventory level starts at state $i$. Solving equations (3) for $\tilde{f}_{i}(\alpha)$ gives

$$
\tilde{f}_{i}(\alpha)=\left(\frac{1-\sqrt{1-4 \tilde{g}(\alpha) \tilde{h}(\alpha)}}{2 \tilde{h}(\alpha)}\right)^{i+1}, i \geq 0
$$

Combining (4) and (1) it can be shown (see Van der Laan, 2003) that the linearization of $A S\left(Q_{p}\right)$ is written as

$$
\overline{A S}\left(Q_{p}\right)=c_{r} u+\left[c_{p}(d-u)+\left(K_{p}+a\right)\left(\frac{d-u}{Q_{p}}\right)+\alpha c_{p}\left(\frac{Q_{p}-1}{2}+\frac{u}{d-u}\right)+b\right] \tilde{f}_{I_{0}}(\alpha)
$$


with $a=\alpha K_{p}\left(\frac{d+u}{2(d-u)^{2}}\right)$ and $b=\alpha\left(c_{p}+K_{p} / 2\right)$. We may interpret $a$ as the (relevant) opportunity cost per batch of a production order.

Note that $\tilde{f}_{I_{0}}(\alpha)$ is a constant, so it is left out of the linearization. The traditional average cost approach computes the average cost function as

$$
A C\left(Q_{p}\right)=c_{r} u+c_{p}(d-u)+K_{p}\left(\frac{d-u}{Q_{p}}\right)+h_{s}\left(\frac{Q_{p}-1}{2}+\frac{u}{d-u}\right) .
$$

The first three terms correspond to the average variable production cost, the average variable remanufacturing cost, and the average ordering cost for production, respectively. The last term is the average serviceable inventory (see e.g. Muckstadt and Isaac, 1981) times the serviceable holding cost parameter $h_{s}$. From the traditional average cost point of view, it is not immediately clear what the value of $h_{s}$ should be. The interpretation that opportunity costs of holding inventories are proportional to the average inventory investment suggests that $h_{s}$ should be set to $\alpha\left(\frac{d-u}{d} c_{p}+\frac{u}{d} c_{r}\right)$, since the inventory of serviceable products is a mixture of produced (fraction $\frac{d-u}{d}$ ) and remanufactured (fraction $\left.\frac{u}{d}\right)$ products with different marginal $\operatorname{costs} c_{p}$ and $c_{r}$, respectively.

However, a comparison of the (approximately) optimal order quantities

$$
Q_{p}^{\overline{A S}}=\sqrt{\frac{2\left(K_{p}+a\right)(d-u)}{\alpha c_{p}}}
$$

and

$$
Q_{p}^{A C}=\sqrt{\frac{2 K_{p}(d-u)}{h_{s}}}
$$

which can easily be obtained by putting the derivative of (5) and (6), respectively, with respect to $Q_{p}$ equal to zero and solving for $Q_{p}$, shows that choosing $h_{s}=\alpha c_{p}$ (independent of $c_{r}$ !) results in similar optimal ordering quantities for both approaches. Note that for moderate values of $u$, the influence of $a$ is limited. Van der Laan (2003) shows that the linearization is very accurate for moderate return probabilities $(<0.8)$. Using the linearization for optimization gives very good performance even for return rates that are close to the demand rate.

We end this section with a simple example that illustrates the importance of setting the right holding cost rate for the $\mathrm{AC}$ approach. The yearly demand rate is $d=100$ and the yearly return rate is $u=80$. Production is much more expensive than remanufacturing $\left(c_{p}=5, c_{r}=1\right)$. The set-up cost for production is $K_{p}=10$. The yearly discount rate is $20 \%(\alpha=0.20)$. If we set $h_{s}=\alpha c_{p}=1$, then $Q_{p}^{A C}=20$, which is close to $Q_{p}^{\overline{A S}}=20.2$. But if we set $h_{s}=\alpha\left(\frac{d-u}{d} c_{p}+\frac{u}{d} c_{r}\right)=1.8$, then the resulting order quantity $Q_{p}^{A C}=33.3$ is far from optimal. 


\subsection{A deterministic system with production, remanufacturing, and disposal}

In this section, we would like to illustrate the complications that arise if we allow for batch remanufacturing and disposal. Unfortunately, the stochastic approach of the previous section is too difficult to apply in that situation, so instead we take a deterministic approach. We remark that it is common (in forward logistics systems) to calculate order quantities using a deterministic model. The resulting order quantities are generally nearoptimal for the corresponding stochastic model also. The model that we will consider is similar to those discussed in Chapter 7. The main difference is that our model includes a set-up cost for disposal, and therefore we analyze strategies with batch disposal.

Consider the model of the previous section, but instead of remanufacturing all product returns upon arrival, we collect returns during a time interval $T$, i.e., we collect $u T$ products. At the end of that interval, we remanufacture $Q_{r}=r T$ and dispose of the rest (batch disposal because there is a set-up cost for disposal). Here, $r \leq u$ may be interpreted as the recovery rate. So, apart from the decision variable $Q_{p}$, we also have the recovery rate $r$ as a decision variable. There are fixed costs $K_{r}$ and $K_{w}$ associated with remanufacturing and disposal respectively. The unit 'cost' related to disposal, $c_{w}$ can be either positive (for instance, if products contain hazardous materials which need to be processed in an environmentally friendly manner) or negative (for instance, if product returns have a positive salvage value and can be sold to a third party). For ease of explanation, we assume here that the inventory system is controlled by repeatedly producing one production batch of size $Q_{p}$, succeeded by one remanufacturing batch of size $Q_{r}$. However, the analysis is easily extended to arbitrary sequences of production and remanufacturing batches. Figure 2 shows the inventory processes involved. Figure 3 shows the associated cash flows. See also Chapter 7, where this type of lot-sizing strategy for a deterministic system is discussed. We make a distinction between disposable inventory and remanufacturable inventory, because the associated cash flows differ. By the same argument, we do not distinguish between produced and remanufactured products because they represent the same value with respect to sales price.

Note that the decision variables, $Q_{p}, Q_{r}$, and $r$, are mutually dependent through the relation $Q_{r}=\frac{r}{d-r} Q_{p}$. We assume that at time 0 we start with zero inventory of both serviceables and remanufacturables. Thus, to start up the system and to guarantee a monotonous ordering strategy at the same time, we have to start with a production batch of size $Q_{r}$. The first regular production batch of size $Q_{p}$ then occurs at time $T_{p}=Q_{r} / d$ and the first remanufacturing batch occurs at time $T=\left(Q_{p}+Q_{r}\right) / d$. Continuing this way, production batches and remanufacturing batches occur every $T$ time units. We remark 

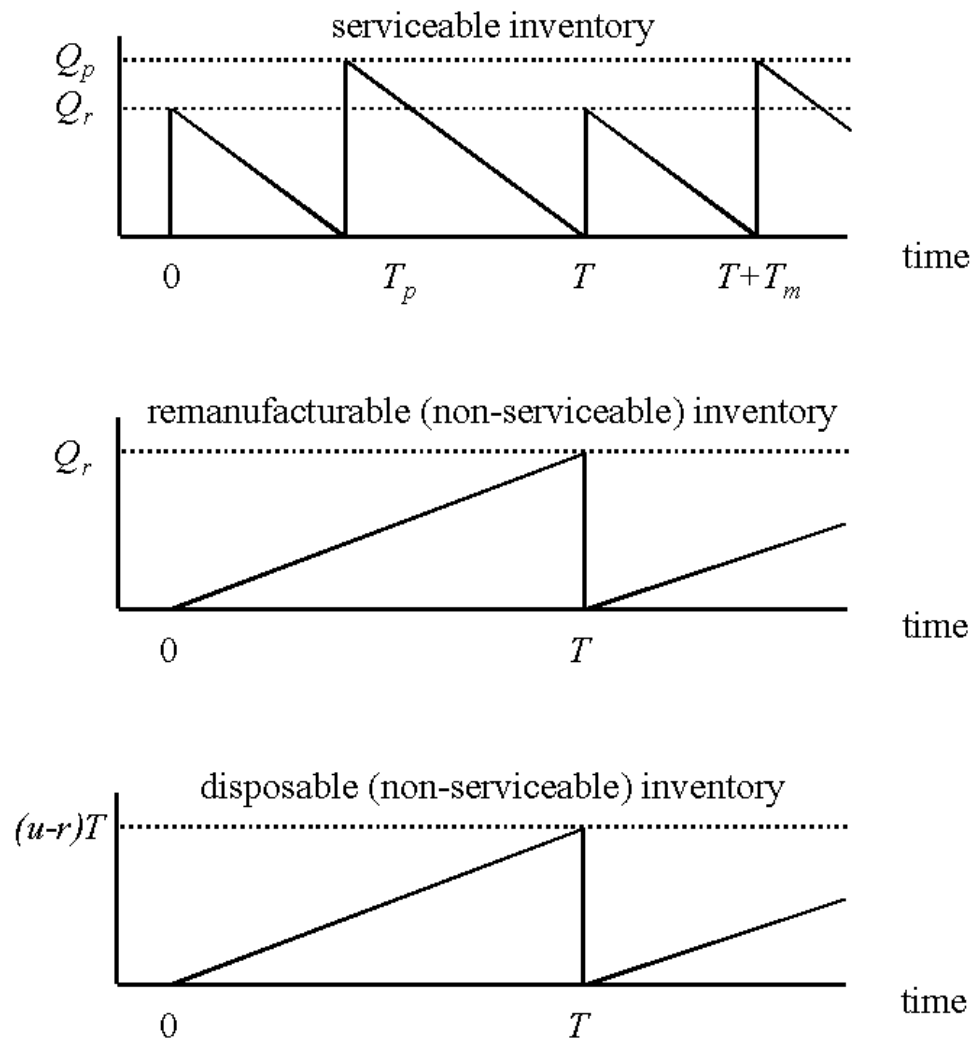

Figure 2: The inventory processes for the system with production, remanufacturing, and disposal

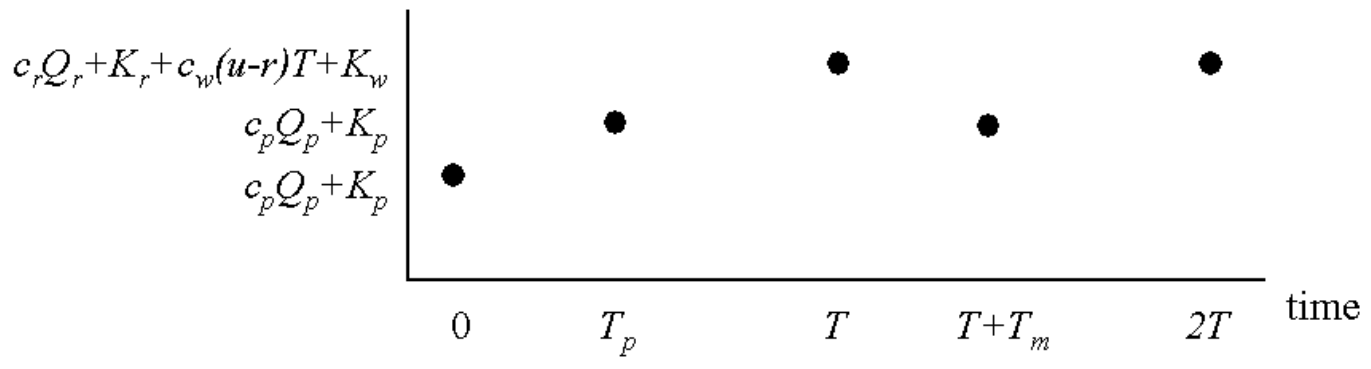

Figure 3: Relevant cash flows for the system with production, remanufacturing, and disposal 
that it would not be 'fair' to compare strategies with different initial inventories, and hence the start-up batch is needed. Lead times are assumed to be zero.

In general, if $T$ denotes the cycling time of a discrete cash flow $C$, with first occurrence time $T_{1}$, then the annuity stream is given by

$$
A S=\alpha C \sum_{n=0}^{\infty} \mathrm{e}^{-\alpha\left(T_{1}+n T\right)}=\frac{\alpha C \mathrm{e}^{-\alpha T_{1}}}{1-\mathrm{e}^{-\alpha T}}
$$

and its linearization in $\alpha$ (using a Tayler series approximation) by

$$
\overline{A S}=\frac{C}{T}+\alpha C\left(\frac{1}{2}-\frac{T_{1}}{T}\right)
$$

Using those results and choosing $C$ and $T_{1}$ according to the cash flows in Figure 3 , we find that for $0 \leq r \leq u<d$, the total annuity stream is given by

$$
A S=\alpha\left(K_{p}+Q_{r} c_{p}+\frac{\left(K_{p}+Q_{p} c_{p}\right) \mathrm{e}^{-\alpha T_{p}}+\left(K_{r}+K_{w}+Q_{r} c_{r}+(u-r) T c_{w}\right) \mathrm{e}^{-\alpha T}}{1-\mathrm{e}^{-\alpha T}}\right)
$$

which can be approximated by the function

$$
\begin{aligned}
\overline{A S}= & c_{p}(d-r)+c_{r} r+c_{w}(u-r) \\
& +(d-r) K_{p} / Q_{p}+r\left(K_{r}+K_{w}\right) / Q_{r}+\alpha K_{p}-\alpha\left(K_{r}+K_{w}\right) / 2 \\
& +\alpha c_{p} Q_{r}+\alpha\left(K_{p}+c_{p} Q_{p}\right)\left(\frac{1}{2}-\frac{T_{p}}{T}\right)-\alpha c_{r} Q_{r} / 2-\alpha c_{w}(u-r) T / 2 \\
= & c_{p}(d-r)+c_{r} r+c_{w}(u-r) \\
& +(d-r) K_{p} / Q_{p}+r\left(K_{r}+K_{w}\right) / Q_{r}+\alpha K_{p}-\alpha\left(K_{r}+K_{w}\right) / 2 \\
& +\alpha\left(K_{p}+c_{p} Q_{p}\right)\left(\frac{1}{2}-\frac{r}{d}\right)+\alpha\left(c_{p}-c_{r} / 2\right) Q_{r}-\alpha c_{w}\left(\frac{u}{r}-1\right) Q_{r} / 2 .
\end{aligned}
$$

Next, we apply the traditional AC approach. Clearly, the numbers of produced, recovered, and disposed items per time unit are $d-r, r$, and $u-r$, respectively. The fractions of demand satisfied by production and recovery are $(d-r) / d$ and $r / d$, respectively. Combining this with Figure 2 and using $Q_{r}=r T$ gives a total average cost of

$$
\begin{aligned}
A C= & c_{p}(d-r)+c_{r} r+c_{w}(u-r) \\
& +(d-r) K_{p} / Q_{p}+r\left(K_{r}+K_{w}\right) / Q_{r} \\
& +h_{s}\left[\left(1-\frac{r}{d}\right) Q_{p} / 2+\left(\frac{r}{d}\right) Q_{r} / 2\right]+h_{r} Q_{r} / 2+h_{w}\left(\frac{u}{r}-1\right) Q_{r} / 2,
\end{aligned}
$$

where $h_{s}, h_{r}$, and $h_{w}$ are the holding cost rates for serviceables, remanufacturables, and disposables, respectively. Using the Relation $Q_{r}=\frac{r}{d-r} Q_{p}$, it is easily verified that we can transform AC into $\overline{A S}$ (up to a constant) by using the following transformation of $c_{p}, h_{s}$, $h_{r}$, and $h_{w}$. 


$$
\begin{aligned}
& c_{p} \rightarrow c_{p}+\alpha K_{p} / d \\
& h_{s} \rightarrow \alpha c_{p} \\
& h_{r} \rightarrow \alpha\left(c_{p}-c_{r}\right) \\
& h_{w} \rightarrow-\alpha c_{w}
\end{aligned}
$$

This transformation is unique with respect to $h_{s}, h_{r}$, and $h_{w}$. Note the value of $h_{s}$ is consistent with the results of the previous section. Furthermore, in the special case that nothing is disposed of, i.e. $r=u$, the value of $h_{r}$ has been validated for several deterministic models (see van der Laan and Teunter, 2002) and stochastic models, as well (Teunter, 2002, Teunter et al., 2000, van der Laan, 2003). The next section attempts to give an intuitive explanation of the above results.

\section{$3 \quad$ Intuitive explanations}

In the previous section, the average cost per time unit and the annuity stream of the total discounted cost (net present value) were compared analytically for some simple models. A set of holding cost rates for the average cost expression was sought that approximately transforms it into the annuity stream of the discounted cost expression, or, for short, that approximately transforms the average cost into the discounted cost. For ease of notation, we will refer to those rates as transformation rates in what follows. The corresponding values, calculated by first subtracting the out-of-pocket holding cost rate and then dividing by the discount rate, will be referred to as the transformation values.

Recall that all analytical studies were based on the inventory system as depicted in Figure 1. Some studies considered strategies that remanufacture all returned products, but others considered strategies that also use the disposal option. For all models that were considered, it turned out that the transformation rates are as follows.

$$
\begin{aligned}
h_{s} & =\alpha c_{p} \\
h_{r} & =\alpha\left(c_{p}-c_{r}\right) \\
h_{w} & =-\alpha c_{w}
\end{aligned}
$$

Note that there are different holding cost rates for remanufacturable returned products and disposable returned products, though these products are identical. Also note that all serviceable items, whether produced or remanufactured, have the same holding cost rate. These two aspects of the transformation rates are not in agreement with the traditional 'cost price reasoning' usually applied to find the holding cost rates for average cost inventory systems without product recovery. That reasoning would lead to identical holding cost rates for disposables and remanufacturables (since their collection cost is identical), but different holding cost rates for produced and remanufactured products (since different 
costs are involved). From a 'cash flow point of view', however, the observed two aspects of the transformation rates do make sense. Disposables and remanufacturables have different future cash flows and hence tie up different amounts of capital, but produced and remanufactured products do not. Below, we will continue this reasoning to intuitively derive values and the corresponding holding cost rates.

First consider production and remanufacturing of serviceable products. The capital tied up in a produced product is $c_{p}$, and so its value is $c_{p}$. A remanufactured product has the same quality as a produced product and is used to satisfy the same demands, so its value is also $c_{p}$. The cost of remanufacturing a product is $c_{r}$, after which a remanufactured product with value $c_{p}$ is obtained. So the value of a remanufacturable item is $c_{p}-c_{r}$. This reasoning leads to the following holding cost rates: $h_{r}=\alpha\left(c_{p}-c_{r}\right), h_{s}=\alpha c_{p}$. See also Teunter, et al. (2000) (they use different notations). Note that these are the transformation rates in (9).

Next consider disposal. The disposal cost is $c_{w}$. So the capital tied up in a disposable item is $-c_{w}$, and hence its value is $-c_{w}$. We remark that $c_{w}$ can be negative and hence the value positive if, for instance, disposal means the lucrative selling of remanufacturable products to a broker. If $c_{w}$ is positive, however, then the value of a remanufacturable product is negative. Indeed, in such a case a remanufacturable product is a liability rather than an asset. This reasoning leads to $h_{w}=\alpha\left(-c_{w}\right)$. See also Teunter et al. (2000). Again, this is the transformation rate in (9).

The analytical results of the previous section and the above reasoning provide some confidence that the holding cost rates in (9) roughly transform an average cost model into a discounted cost model (for the system depicted in Figure 1). The simulation results of Teunter (2002) provide some additional evidence. He assumes that both demand and return are stochastic (driven by Poisson processes). He analyzes EOQ-strategies with fixed order quantities for production and remanufacturing (no disposal). These strategies imply that a new batch should be ordered if the stock drops below 0 (zero lead times); a remanufacturing batch is ordered if enough remanufacturable products are available and a production batch is ordered otherwise. For all the examples that he considers, the average cost model with holding cost rates as in (9) and the discounted cost model lead to approximately the same (simulated) optimal order quantities.

But the analytical results and their intuitive explanation also show the limitations of the average cost approach. The transformation rates (9) can only be applied if returned products are marked as either disposable (i.e. to be disposed of) or remanufacturable (i.e. to be remanufactured) directly upon arrival. In situations with stochastic demand and return processes, however, it seems better to apply inventory strategies that base remanufacturing and disposal decisions on the serviceable inventory level. See e.g. van der Laan et al. (1999). In such situations where a distinction between disposables and remanufacturables can not be made, the same holding cost rate has to be used for both. It seems reasonable to then use some mixture of $\alpha\left(c_{p}-c_{r}\right)$ and $-\alpha c_{w}$, but that will in general lead to sub-optimal remanufacturing decisions as well as sub-optimal disposal decisions (see also van der Laan and Teunter, 2002). 


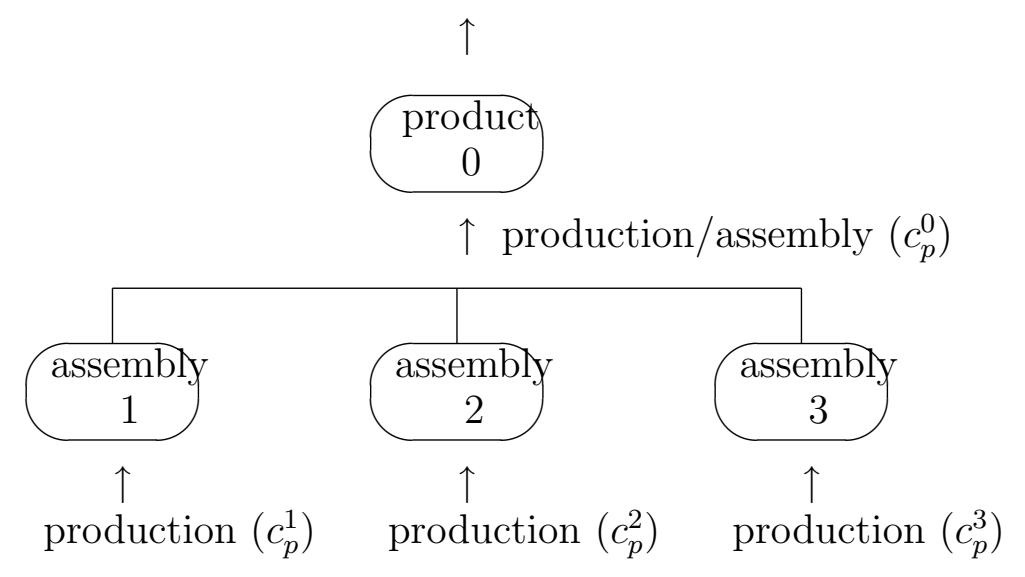

Figure 4: Example of a product structure and corresponding production costs

In the next section, we try to generalize our findings for more complex inventory systems with disassembly of returned products.

\section{Systems with disassembly}

So far, we have restricted ourselves to the inventory system depicted in Figure 1. In this section, we consider more complex systems with disassembly of returned products. Most of the material presented in this section is based on Teunter (2001).

Our discussion will be for inventory systems with product (dis)assembly in general, but we will illustrate it using the example of a product that consists of three components (no subdivision of components into parts, etc.). The product structure and corresponding production costs are depicted in Figure 4. For ease of notation, we will refer to products as well as components, parts, etc., as assemblies.

Based on the results/arguments of the previous sections, we expect that the average cost approach is not capable of (roughly) including capital costs if different recovery/disposal options are used for one or more assemblies. Assume, for instance, that the product in Figure 4 is disposed of in some cases but disassembled into its three components (which are then recovered) in other cases. The decision to dispose or disassemble could depend on the stocks of remanufacturable and produced/remanufactured serviceable components. Since there are different costs and revenues associated with the disposal option and the disassembly option, there will not be a single set of values (and corresponding holding cost rates) that approximately transforms the average cost into the discounted cost.

But we do expect that a set of transformation values exists if the recovery strategy is fixed. By recovery strategy we mean a description of the (partial) disassembly scheme and of the recovery/disposal operations for all disassembled assemblies. An example of 


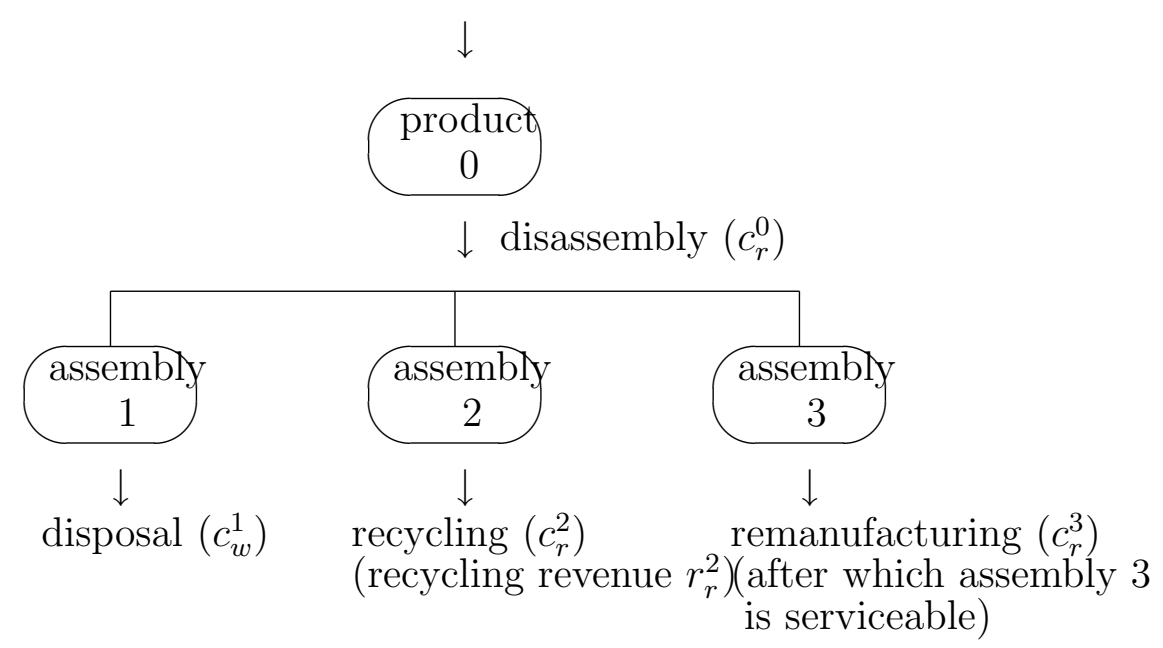

Figure 5: A recovery strategy for the product of Figure 4 and the associated recovery/disposal costs. For assembly 2 , the recycling revenues, i.e. the value of the obtained materials, is also given.

\begin{tabular}{l|llll} 
assembly & production cost & disposal cost & recovery cost & recovery revenue \\
\hline 0 & $c_{p}^{0}$ & & $c_{r}^{0}$ (disassembly) & \\
1 & $c_{p}^{1}$ & $c_{w}^{1}$ & $c_{r}^{2}$ (recycling) & $r_{r}^{2}$ \\
2 & $c_{p}^{2}$ & & $c_{r}^{3}$ (remanufacturing) &
\end{tabular}

Table 2: Notations introduced in Figures 4 and 5

a fixed recovery strategy for the product of Figure 4 and the associated costs/revenues are given in Figure 5. This is just one possible recovery strategy and not necessarily the optimal one. We refer readers who are interested in the determination of the optimal recovery strategy to Chapter 10. The notations that have been introduced in Figures 4 and 5 are listed in Table 2.

If the recovery strategy is fixed, then the costs/revenues associated with the recovery/disposal are also fixed, and hence the values are fixed. Based on the results in previous sections, the following valuation method is proposed (see Teunter, 2001).

\section{Valuation Method}

The value of a produced or an as-good-as-new remanufactured assembly is the production cost (price). The value of a recoverable/disposable assembly is its net profit. The net profit is equal to revenue minus cost, where the revenue of remanufacturing is the value of a remanufactured assembly.

We will illustrate this method for the product structure depicted in Figure 4 and the recovery strategy depicted in Figure 5. For further discussion on how to determine the 


\begin{tabular}{c|ll} 
& \multicolumn{2}{|c}{ value of assembly } \\
assembly & produced/remanufactured & recoverable \\
\hline 1 & $c_{p}^{1}$ & $-c_{w}^{1}$ \\
2 & $c_{p}^{2}$ & $r_{r}^{2}-c_{r}^{2}$ \\
3 & $c_{p}^{3}$ & $c_{p}^{3}-c_{r}^{3}$ \\
0 & $c_{p}^{0}+c_{p}^{1}+c_{p}^{2}+c_{p}^{3}$ & $-c_{r}^{0}+\left(-c_{w}^{1}\right)+\left(r_{r}^{2}-c_{r}^{2}\right)+\left(c_{p}^{3}-c_{r}^{3}\right)$
\end{tabular}

Table 3: Values for recoverable and recovered assemblies associated with the product structure in Figure 4 and the recovery strategy presented in Figure 5, resulting from the proposed valuation method

net profits of recoverable/disposable assemblies, we refer interested readers to Krikke et al., (1998).

Using Figure 4, the values for produced/recovered assemblies can easily be calculated starting with the components (bottom-up, in general). Similarly, given those values, Figure 5 can be used to calculate the net profits of recoverable/disposable assemblies, again starting with the components (bottom-up, in general). The calculations are done in Table 3.

For the simple inventory system depicted in Figure 1 without the disposal option, the proposed valuation method indeed results in the values and corresponding holding cost rates $\left(h_{n}=\alpha\left(c_{p}-c_{r}\right), h_{r}=h_{p}=\alpha c_{p}\right)$ that were shown in Section 2 to roughly transform the average cost into the discounted cost. Unfortunately, it is not possible to analytically test the valuation method for complex inventory systems with (dis)assembly.

Teunter (2001) tests the valuation method for an inventory system with 3 levels (one product, two components, four parts) using simulation. For all examples that he considers, it turns out that the optimal (cost minimizing) strategies under the average cost approach (combined with the valuation method) and the discounted cost approach are (almost) identical. This provides some confidence that the valuation method indeed works. We do remark that only a single recovery strategy is considered and that all production and recovery lead times are assumed to be zero. The zero lead times allow a restricted focus on inventory strategies that are characterized by batch sizes alone, making the search for the optimal strategy manageable.

Of course, more (simulations) tests are needed before we can be confident that the proposed valuation method works in general. Performing such tests will be time-consuming, since it involves the simultaneous determination of inventory strategies for all assemblies of a product structure. But the effort is certainly worthwhile. Knowing the way to roughly include capital costs in an average cost model of a product recovery inventory system implies that the use of a more difficult discounted cost system can be avoided. 


\section{Implications for multiple-source systems}

The previous sections have shown that a major complicating factor for valuing inventories in systems with product recovery is the two-source character of those systems. Production and recovery have different associated marginal costs. As a result of this cost difference, it is not straightforward to determine the 'right' value (and the corresponding holding cost rates) for a serviceable product. In fact, the reported findings indicate that the 'traditional' cost-price reasoning should not be used for systems with recovery. In this section, we discuss the implications for multiple-source systems in general.

There are several reasons for using multiple sources in systems without product recovery: e.g. capacity restrictions of the source with the lowest cost (or price), a large lead time for the source with the lowest cost (regular and emergency ordering), or the reduced lead time that results from order-splitting. See also Fearon (1993) for a discussion of advantages and disadvantages of multiple sourcing versus single sourcing.

Multiple source models have been studied by many authors, e.g. Hong and Hayya (1992), Lau and Zhao (1993), and Sculli and Wu (1981). However, to the best of our knowledge, none of them address the problem of determining the correct inventory value of an arbitrary product. They either assume the value to be fixed and given, or calculate it as a (weighted) average of the marginal costs for the different sources (i.e. the average cost price).

Based on the reported finding for product recovery systems, we expect that the simple approach of averaging marginal costs is not correct from a DCF point of view. However, the approach seems reasonable as long as the differences in marginal costs are small (say less than 10\%). For many multiple-source systems, the cost differences will indeed be small, since sources will not be used if they are too expensive. This partly explains why the issue of inventory valuation has not been addressed in the literature. However, for models with a regular and an emergency replenishment option, the cost difference can be large (even more than 100\%). So especially for these models, future research is needed on valuing inventories.

\section{Conclusions and directions for further research}

Values of recoverable and recovered products (and components, parts, etc.) are relevant for accounting purposes and also for strategic and operations management decisions. In this chapter, we discussed their relevance for inventory control decisions. Inventory control is linked to product valuation through holding cost rates. For average cost (AC) models, which dominate inventory control, it is common use to include product values (multiplied by an interest rate) in the holding cost rates, thereby roughly correcting the mistake of having not discounted cash flows properly.

In Section 2, the average cost and the (annuity stream of the) discounted cash flow were compared analytically for some simple models. These models are all based on a simple inventory system, where returned products can either be remanufactured or disposed of. 
They differ in the type of inventory strategy considered. It appeared that transformation rates, i.e. a set of holding cost rates that approximately transforms the average cost into the discounted cash flow, indeed exist (for the considered models). Moreover, we observed that these rates are very different than those that would result from applying the traditional cost-price logic. For instance, that logic would result in equal holding cost rates for all returned non-serviceable products, but it turns out that different rates should be used for remanufacturables and disposables.

In Section 3, we offered intuitive cash flow explanations for the transformation rates. In short, remanufacturing and disposal have different associated revenues/costs, and hence lead to different values of returned products. Unfortunately, this implies that in order to know the right $(\mathrm{AC})$ holding cost rate of a returned product, one has to know whether that product will be disposed of or remanufactured. This is not necessarily true for any inventory model, and we therefore discussed the limitations of using the transformation rates that were derived in Section 2.

Sections 2 and 3 only considered the simple inventory model where a returned product is either directly disposed of or directly remanufactured. In Section 4, more complex inventory systems with product (dis)assembly were considered. We proposed a method for determined transformation rates and discussed its limitations (the recovery strategy is fixed).

Testing the proposed valuation method is one area for further research. Other research directions are to analyze the effect of (returned) product values on strategic and operations management decisions other than inventory decisions, and to perform case studies. Finally, inventory valuation should be studied for more general multiple-source systems, especially systems with a regular and an emergency supply option (see Section 5).

\section{References}

[1] M. Corbey, K. Inderfurth, E. van der Laan, and S. Minner. The use of accounting information in production and inventory control for reverse logistics. submitted to Management Accounting Research, 1999.

[2] H.E. Fearon and M.R. Leenders. Purchasing and Materials Management. Irwin, Homewood Ill., 1993.

[3] M. Fleischmann and R. Kuik. On optimal inventory control with stochastic item returns. Management Report 21-1998, Erasmus University Rotterdam, 1998.

[4] J.D. Hong and J.C. Hayya. Just-in-time purchasing: single or multiple sourcing? International Journal of Production Economics, 27:175-181, 1992.

[5] W.K. Klein Haneveld and R.H. Teunter. Effects of discounting and demand rate variability on the EOQ. International Journal of Production Economics, 54:173-192, 1998. 
[6] H.R. Krikke, A. van Harten, and P.C. Schuur. On a medium term product recovery and disposal strategy for durable assembly products. International Journal of Production Research, 36(1):111-139, 1998.

[7] H.S. Lau and L.G. Zhao. Optimal ordeing with two suppliers when lead times and demand are all stochastic. European Journal of Operational Research, 68:120-133, 1993.

[8] J.A. Muckstadt and M.H. Isaac. An analysis of single item inventory systems with returns. Naval Research Logistics Quarterly, 28:237-254, 1981.

[9] E. Naddor. Inventory Systems. John Wiley and Sons, New York, 1966.

[10] D. Sculli and S.Y. Wu. Stock control with two suppliers and normal lead times. Journal of Operational Research Society, 32:1003-1009, 1981.

[11] E.A. Silver, D.F. Pyke, and R. Peterson. Inventory Management and Production Planning and Scheduling. John Wiley and Sons, New York, 1998.

[12] R.J. Tersine. Principles of Inventory and Materials Management. Elsevier Science Publishing Company, New York, 1988.

[13] R.H. Teunter. A reverse logistics valuation method for inventory control. International Journal of Production Research, 39(9):2023-2035, 2001.

[14] R.H. Teunter. Economic ordering quantities for stochastic discounted cost inventory systems with remanufacturing. International Journal of Logistics, forthcoming, 2002.

[15] R.H. Teunter, E.A. van der Laan, and K. Inderfurth. How to set the holding cost rates in average cost inventory models with reverse logistics? OMEGA The International Journal of Management Science, 28:409-415, 2000.

[16] E. van der Laan. An NPV and AC analysis of a stochastic inventory system with joint manufacturing and remanufacturing. ERIM report series 2000-38-LIS, 2000.

[17] E.A. van der Laan, M. Salomon, R. Dekker, and L.N. van Wassenhove. Inventory control in hybrid systems with remanufacturing. Management Science, 45(5):733$747,1999$.

[18] E.A. van der Laan and R.H. Teunter. Average costs versus net present value. In Quanitative approaches to distribution logistics and supply chain management. Lecture notes in economics and mathematical systems, Springer-Verlag, 2002. 